\title{
Commentary Can fuzzy logic make things more clear?
} Jan A Hazelzet

Pediatric ICU, Erasmus MC, Sophia, 3000CB, Rotterdam, The Netherlands

Corresponding author: Jan A Hazelzet, j.a.hazelzet@erasmusmc.nl

Published: 18 February 2009

Critical Care 2009, 13:116 (doi:10.1186/cc7692)

This article is online at http://ccforum.com/content/13/1/116

(C) 2009 BioMed Central Ltd

See related research by Merouani et al., http://ccforum.com/content/12/6/R155

\begin{abstract}
Intensive care is a complex environment involving many signals, data and observations. Clinical decision support and artificial intelligence using fuzzy logic and closed loop techniques are methods that might help us to handle this complexity in a safe, effective and efficient way. Merouani and colleagues have performed a study using fuzzy logic and closed loop techniques to more effectively wean patients with sepsis from norepinephrine infusion.
\end{abstract}

In the intensive care environment clinicians are trying, surrounded by a wealth of information and using guidelines as well as their personal expertise, to treat patients in the best possible way. We know that these treatments are complex and not always consistent among clinicians. They might also be influenced by a number of other conditions, such as workload, and human conditions, such as fatigue or personal feelings and intuition. Examples of these treatments are mechanical ventilation or multidrug hemodynamic support in septic shock. Not only is the commencement of these treatments important, but also the weaning process, in order to limit possible side effects resulting from them.

Weaning from vasopressors is often approached empirically and performed manually. Bedside equipment such as pressure transducers, infusion pumps, pulse oximeters and mechanical ventilators store their data in clinical information systems. Artificial intelligence tools can function as intelligent assistants to clinicians, constantly monitoring data for trends or adjusting the settings of devices. In a recent issue of Critical Care, Merouani and colleagues [1] describe a completely automated weaning protocol based on closed loop control using fuzzy logic principles. They could show a reduction in the duration of norepinephrine weaning in septic patients enrolled in this automated protocol study group in comparison to a control group where the weaning occurred at the clinician's discretion. Also, the total amount of norpinephrine administered was significantly reduced in the automated group compared with the control group.

What is fuzzy logic? Medical biological processes can be so complex and unpredictable that physicians sometimes must make decisions based on intuition. Computers are capable of making calculations at high and constant speed and of recalling large amounts of data and can, therefore, be used to manage decision networks of high complexity. However, binary, or 'crisp', logic, situations arising from medical biological processes are difficult for them to handle. Fuzzy logic, on the other hand, is a form of multi-valued logic that deals with reasoning that is approximate rather than precise. For instance, in the case of population height where the average height is $1.8 \mathrm{~m}$, binary, or 'crisp', logic would determine a person of $1.79 \mathrm{~m}$ to be of medium height, and other people who are, for example, $1.81 \mathrm{~m}$ or $2.25 \mathrm{~m}$ would be considered tall. In fuzzy logic, however, there are no such heights as $1.83 \mathrm{~m}$, but only fuzzy values such as dwarf, small, medium, tall, giant. The highest values belonging to the set 'dwarf' can overlap with the lowest values of the set 'small'. While variables in mathematics usually take numerical values, in fuzzy logic applications non-numeric linguistic variables are often used to facilitate the expression of rules and facts. Thus, fuzzy logic has a particular advantage in areas where precise mathematical description of control processes is impossible and is thus especially suited for use in supporting medical decision making [2-4]. Other examples of described systems where closed loop fuzzy logic techniques have been used include mechanical ventilation [5,6], anesthesia [7-9], neurosurgery and intracranial pressure monitoring [9-11].

In their study, Merouani and colleagues measured mean arterial pressure (MAP) every 10 seconds for 7 minutes to obtain an accurate MAP measurement with the least possible number of artifacts and then processed all obtained values with median values filtering. A computer converted the MAP

$\mathrm{MAP}=$ mean arterial pressure 
and norepinephrine infusion rate into fuzzy datasets and automatically calculated the required change in rate of infusion. MAP level and MAP variation ( $\triangle M A P)$, the variables to be controlled, were the outputs of the controlled system, whereas the norepinephrine infusion rate was the input to be adjusted to reach the desired MAP value. This makes it a closed loop control system. The infusion rate changed automatically every 7 minutes after analysis of the MAP and the $\triangle M A P$. The timeframe of infusion rate modifications was empirically set at 7 minutes in order to take into account the equipment's inertia and patient's time to hemodynamic response. The results in this study are promising, although the study does not dedicate much attention to the side effects or safety issues of this kind of technique. For this study, a study manager was available constantly, but it was not reported how frequent this person had to be consulted. To be useful, such systems should be designed to be effective, safe, and easy to use at the bedside. In particular, these systems must be capable of noise removal, artifact detection and effective validation of data [5].

Fuzzy logic provides a means for encapsulating the subjective decision making process in an algorithm suitable for computer implementation. More research is necessary to develop fuzzy logic algorithms for certain medical processes, followed by safety testing and, eventually, validation in patients [2,12]. This will support the management of complex treatments in the intensive care unit, reduce variability between physicians and help us in achieving clinical endpoints.

\section{Competing interests}

The author declares that they have no competing interests.

\section{References}

1. Merouani M, Guignard B, Vincent F, Borron SW, Karoubi P, Fosse JP, Cohen Y, Clec'h C, Vicaut E, Marbeuf-Gueye C, Lapostolle F, Adnet F: Norepinephrine weaning in septic shock patients by closed loop control based on fuzzy logic. Crit Care 2008, 12: R155.

2. Bates JH, Young MP: Applying fuzzy logic to medical decision making in the intensive care unit. Am J Respir Crit Care Med 2003, 167:948-952.

3. Hanson CW 3rd, Marshall BE: Artificial intelligence applications in the intensive care unit. Crit Care Med 2001, 29:427-435.

4. Christensen M, Hewitt-Taylor J: Defining the expert ICU nurse. Intensive Crit Care Nurs 2006, 22:301-307.

5. Tehrani FT, Roum JH: Intelligent decision support systems for mechanical ventilation. Artif Intell Med 2008, 44:171-182.

6. Belal SY, Taktak AF, Nevill A, Spencer A: An intelligent ventilation and oxygenation management system in neonatal intensive care using fuzzy trend template fitting. Physiol Meas 2005, 26:555-570.

7. Puri GD, Kumar B, Aveek J: Closed-loop anaesthesia delivery system (CLADS) using bispectral index: a performance assessment study. Anaesth Intensive Care 2007, 35:357-362.

8. Haddad WM, Bailey JM, Hayakawa T, Hovakimyan N: Neural network adaptive output feedback control for intensive care unit sedation and intraoperative anesthesia. IEEE Trans Neural Netw 2007, 18:1049-1066.

9. Shieh JS, Fu M, Huang SJ, Kao MC: Comparison of the applicability of rule-based and self-organizing fuzzy logic controllers for sedation control of intracranial pressure pattern in a neurosurgical intensive care unit. IEEE Trans Biomed Eng 2006, 53:1700-1705.
10. Roitberg B: Fuzzy logic in the neurosurgical intensive care unit. Surg Neurol 2006, 65:217.

11. Huang SJ, Shieh JS, Fu M, Kao MC: Fuzzy logic control for intracranial pressure via continuous propofol sedation in a neurosurgical intensive care unit. Med Eng Phys 2006, 28: 639-647.

12. Adlassnig KP, Combi C, Das AK, Keravnou ET, Pozzi G: Temporal representation and reasoning in medicine: Research directions and challenges. Artif Intell Med 2006, 38:101-113. 